\title{
An Empirical Investigation of the Challenges of Cloud-Based ERP Adoption in Pakistani SMEs
}

\author{
Mujtaba Awan (D), ${ }^{1}$ Niamat Ullah $\left(\mathbb{D},{ }^{2}\right.$ Sikandar Ali $\left(\mathbb{D},{ }^{3,4}\right.$ Irshad Ahmed Abbasi $\left(\mathbb{D},{ }^{5}\right.$ \\ Muhammad Shabbir Hassan $\mathbb{D},{ }^{1}$ Hizbullah Khattak $\mathbb{D}{ }^{6}{ }^{6}$ and Jiwei Huang $\mathbb{D}^{3,4}$ \\ ${ }^{1}$ Department of Software Engineering, Riphah International University Islamabad, Rawalpindi, Pakistan \\ ${ }^{2}$ Department of Computer Science, University of Buner, Buner 17290, Pakistan \\ ${ }^{3}$ Department of Computer Science and Technology, China University of Petroleum-Beijing, Beijing 102249, China \\ ${ }^{4}$ Beijing Key Lab of Petroleum Data Mining, China University of Petroleum-Beijing, Beijing 102249, China \\ ${ }^{5}$ Department of Computer Science, Faculty of Science and Arts, Belgarn, P.O. Box 60, Sabt Al-Alaya 61985, \\ University of Bisha, Saudi Arabia \\ ${ }^{6}$ Department of Information Technology, Hazara University Mansehra, Khyber Pakhtunkhwa, Pakistan
}

Correspondence should be addressed to Sikandar Ali; sikandar@cup.edu.cn and Jiwei Huang; huangjw@cup.edu.cn

Received 14 February 2021; Revised 21 March 2021; Accepted 23 March 2021; Published 1 April 2021

Academic Editor: Habib Ullah Khan

Copyright (C) 2021 Mujtaba Awan et al. This is an open access article distributed under the Creative Commons Attribution License, which permits unrestricted use, distribution, and reproduction in any medium, provided the original work is properly cited.

Cloud-based ERP solutions offer many benefits to small and medium enterprises (SMEs) and help them to integrate their activities, such as improve communications and reduce operational and maintenance costs. Primarily, it was only adopted by large organizations, but now SMEs are also keen on adoption. However, the motivation regarding the adoption of these systems in SMEs is relatively low in developing countries. This fact urges us to investigate the challenges faced by Pakistani SMEs. A qualitative research approach along with unstructured interviews was conducted by means of face to face. Interview methods are used to extract understanding, opinions, and challenges faced by SMEs on their way to adopt the cloud-based ERP system. The data were collected from eight well-reputed organizations, directly involved in the adoption. The study found ten (10) themes that are reluctant to adopt cloud ERP among Pakistani SMEs. The main benefit of these themes is to provide results that can be easily accessible to enterprises who want to adopt a cloud-based ERP. This can also contribute to the lack of the literature of cloud ERP and delivers insight for future study by practitioners and researchers.

\section{Introduction}

The most significant and impacting counterpart of the world economy is the business industry, majority of which consists of small and medium enterprises (SMEs). These enterprises are the key enabler for socio-economic development, job creation, poverty reduction, entrepreneurship, and rural development. SME's business environment has changed significantly in recent years for customer satisfaction and market flexibility. Various measures are taken by organizations to meet these challenges such as business model innovation, improved customer service, job automation as well as the development of the IT system such as enterprise resource planning (ERP) $[1,2]$. ERP provides a safe strategy for SMEs in terms of organization operation processes and data management [3]. It is an integrated system known for improving processes and product quality, reducing production cycle time, and enhancing the decision-making process [4]. Despite the recognized significant benefits, traditional ERP is mostly a costly resource [5]. Cloud technology made ERP routed on a cloud platform with lower cost, scalability, and resource sharing [6]. Cloud ERP has grown exponentially in the last few decades due to the new delivery model for ERP, providing various advantages to organizations [7].

In contrast to traditional ERP, cloud ERP offers reduced capital expenditure, rapid execution, increased platforms, and improved storage and data processing. Cloud ERP has 
become an interesting research area among researchers and experts due to its potential advantages to both SMEs and large enterprises [8-10].

\section{Literature Review}

Previous studies have addressed several perspectives while adopting cloud ERP software by SMEs. These include the economical perspective, technological perspective, and the people perspective, which are, respectively, dealt with financial issues, software evaluation, and its effect on people within the organization.

Studies conducted earlier exclusively revealed benefits in implementing cloud ERP in SMEs, such as efficient business processes, real-time access, visibility, accuracy of the information, and effective information management $[11,12]$.

Seethamraju examined the potential determinants using a cross-sectional field study. These include the low total cost of ownership, low investment cost, vendor participation in value cocreation, and product improvement [13].

Salim applied a content analysis method through which they identified 27 transition factors that contribute to the adoption of cloud-based ERP. The study aims to explore which transition factors are important to the different stages of the adoption for the organization to progress to the next level, and these transition factors are defined as "necessary" or "sufficient," where "necessary" transition factors need to occur, while "sufficient" means aiding in the movement [14].

The study reported by Peng et al. explored potential benefits and barriers associated with the adoption of cloudbased ERPs. A set of in-depth interviews are conducted with 16 enterprises and consultants. The results, derived from thematic analysis, showed that while the economic and technological benefits offered by cloud vendors are appealing, the success of cloud-based ERP adoption can be affected by critical challenges related to diverse organizational factors as well as with current legal and technical complexity in the cloud environment [15].

Albar and Hoque conducted ERP investigations in Saudi Arabian context utilizing the TOE framework and DOI theory. Factors identified include the competitive environment, complexity, observability, relative advantage, ICT infrastructure, regulatory environment, ICT skill, and top management support [16].

The practicality of cloud ERP systems for SMEs was further investigated by Zadeh et al. conducting a case study of the USA food industry. Identified benefits include increased performance, agility, flexibility, and cost savings. They also identified that vendor lock-in, security, and compliance issues are the major risk in this practice over the long run [17].

Usman et al. developed the TOE framework and DOI theory to explore determinant factors for cloud ERP adoption in the manufacturing sector of Nigeria. The most influential factors affecting Nigerian SMEs are compatibility, cost savings, lack of data security, competitor's pressure, regulatory support, and cloud ERP knowledge [18].

Ali et al. conducted a study which guided software development organization for cloud-based testing adoption.
The study explores determinants and predictors of cloud adoption for software testing, and a list of predictors (main criteria) and factors (subcriteria) are identified using SLR approach. Seventy subcriteria are identified in this study and also known as influential factors [19].

Moh'd Anwer investigated the main logistical factors that are having impacts on the cloud ERP adoption among SMEs in developing economies of various countries. Overall, 14 factors such as relative advantage, security concerns, compatibility, complexity, value creation, technology readiness, technical barriers, enterprise readiness (ER), enterprise size (ES), enterprise status, top management support (TMS), competitive advantage, government support, and infrastructure/telecommunication were identified using logistic regression analysis [20].

Tongsuksai et al. published an SLR report from 81 articles on cloud ERP implementation. They investigated 32 critical success factors as well as an integrative model based on the organizational, environmental, technological, and individual characteristics. The identified CSFs and factors offer more clarity to (IT) practitioners and help organizations to achieve successful implementation of cloud ERP systems [21].

Ahn and Ahn conducted a comprehensive analysis using DOI theory and TOE frameworks, identifying important influencing factors like trial ability, vendor lock-in, regulatory environment, and relative advantage. This study's findings provide meaningful guidance for cloud-based ERP adoption by companies, agencies supporting enterprise digitalization, and cloud ERP vendors [22].

Ali and Li proposed a study that aims at developing a technology acceptance model. The model provides decision support to software development organizations in various predictors and determinants that will guide organization towards cloud adoption for software testing [23].

The implementation of cloud ERP in business enterprises is a relatively new phenomenon in terms of its influencing factors, inhibitors, and organizational determinants that affect its adoption and management. Understanding the determinants that encounter in the cloud ERP adoption in SMEs helps these organizations to accomplish better results upon their IT investments. Citing the previous literature, it becomes obvious that the main focus of the research in this area is the determinations of challenges and benefits of cloud ERP and factors affecting its adoption in small and medium enterprises. This results in exploring the fact that SMEs are not much prompt to adopt a cloud ERP system. Major investigation upon the low rate of cloud ERP adoption in this side of the business industry has been conducted in the context of developed countries [14].

Literature survey revealed that minimal to no research has been done to inspect the factors influencing the adoption of cloud ERP in developing countries like Pakistan. The present investigations aimed to address the major constraints and challenges faced by Pakistani SMEs while adopting cloud ERP systems. The acceptance and interest rate in the cloud ERP among SMEs are relatively slow and discouraging because of the lack of success stories. Hence, there is a need to identify the factors and determinants as well as other critical influencing factors [24-26]. 
The business practices embedded in the ERP system most likely reflected the US and European organization and their national culture. Upon implementation of such systems in Asian countries, problems may occur due to a mismatch between the cultural assumption and practices embedded in the software. Therefore, the investigations in this area of research will identify the factors that affect the adoption of cloud ERP systems in Pakistani SMEs, which may help the business industry to overcome the major challenges faced in implementing cloud technologies [27].

\section{Methodology}

This section describes the descriptions of the research methodology of the study that includes research questions, research method, data collection techniques, validation, and the detailed processes of the research.

3.1. Research Process. The first level of the research study was the literature review, conducted to understand the available information and data presented by previous researchers. Being an innovative research area, the cloud ERP was previously studied as a cloud and ERP under the titles separately. The second level of the present qualitative study was to conduct interviews from various organizations in Pakistan working on the said concept and was to evaluate different problems faced while adopting a cloud ERP system. The third level of the research was to present the data finding and data analysis [28].

3.2. Data Processing. The major objective of the current study was to investigate the constraints that impact the small and medium enterprises in adopting cloud ERP. The qualitative research methodology was utilized to accomplish the targeted objective as the literature review revealed that cloud ERP is still an innovative and unexplored area of the research as well as very limited empirical studies have been done. The selected methodology also helps to facilitate the researcher towards people understandings regarding the beliefs, behaviors, experience, social, and cultural experiences within the community [29]. Additionally, the proposed research idea aims to explore the field deeply, practicing in the small and medium organizations of Pakistan.

3.3. Data Collection. Data collection is one of the central parts of every research methodology, and hence it is necessary for the researchers to evaluate the previous information carefully and to adopt an appropriate strategy for data collection regarding the available facts. Cloud ERP being an unexplored area technology, the nature of the proposed study was quite complex, requiring a deep understanding of the problem area. For this reason, we decide to collect our empirical data utilizing the concept of the qualitative interview. This method of data collection is one of the professional conversations with precise structure and purpose in which two parties discuss a theme of mutual interest. It is through the interaction between the researcher and the respondent that knowledge is produced [30].

The unstructured interview type was selected to gather the necessary information about the organizations to collect the empirical data. This allowed asking a set of predefined open-ended questions regarding each theme to which the participants could provide clear answers. This approach is quite flexible that did not require any interview guide to follow. It also allows to change the order of the questions to critically follow-up on respondent's answers as well as the further probe and inquire through additional questions. We could therefore keep the interviews open without losing control or direction [31].

3.4. Data Analysis. The process of data analysis starts with transcribing the recorded interviews and translating them into phrases or text to analyze them using the content analysis technique [32]. Qualitative content analysis generally uses individual themes that might be conveyed in a single word, a phrase, a sentence, a paragraph, or an entire document. This technique helps to develop an understanding of collecting data and delivers the ability to test theoretical issues. In the following section, an analysis of the collected data from the Pakistani SMEs on cloud ERP system is presented and discussed. This analytical study emerged ten (10) most important themes which are discussed in the next section in detail.

\section{Results and Discussion}

This section presents the empirical data collected through interviews with various participants to identify the challenges, faced by an organization adopting the cloud ERP solution. The pie chart in Figure 1 shows the overall correlation between consumer concern and identified challenges and the frequency of each challenge/barrier faced by participants within their enterprises. The final discussion of the collected data indicated the suitability and aptness of cloud ERP in Pakistani small and medium organizations as well as future perspectives of the current research study.

4.1. Enterprises. Participants from 8 different organizations who contributed to this research are presented in Table 1.

4.2. Challenges Faced by SMEs. Challenges were identified through the analysis of recorded data. We listen to each interview very carefully again and again and note down important answers and claims by all participants; later on, all these points and claims are translated into common text/ labels and stored in the word document. There were 10 challenges extracted from all participants during the interview session, the percentages and frequencies of the extracted challenges were established and are shown in Figure 1, and the challenges along with their frequencies are shown graphically. 


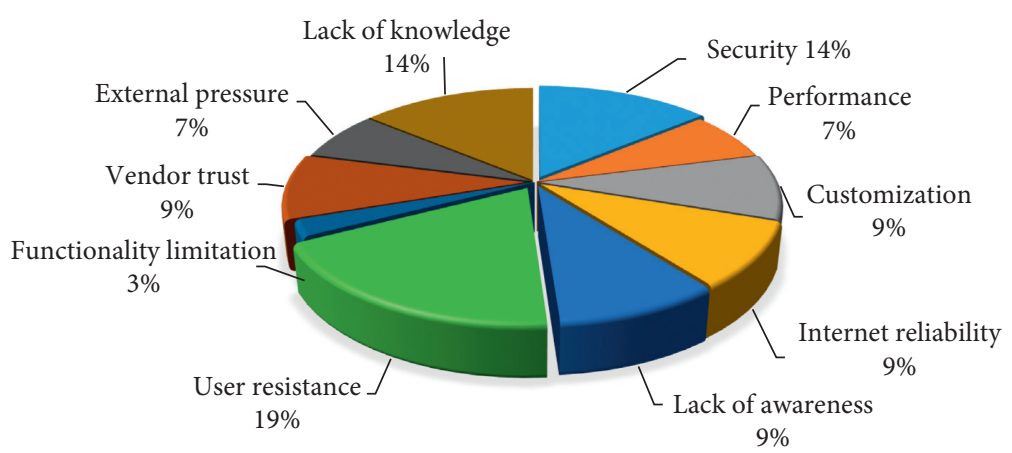

FIgURE 1: Pie chart showing a correlation between consumer concern and identified challenges.

TABLE 1: Overview of conducting interviews.

\begin{tabular}{llcccc}
\hline S.\# & Name & Size & Position & Business location & Duration (min) \\
\hline 1 & Participant 1 & 200 & Manger products & National & 60 \\
2 & Participant 2 & 20 & Director, IT & National & National \\
3 & Participant 3 & 20 & Business analyst & International & 45 \\
4 & Participant 4 & 100 & Manager & National & 50 \\
5 & Participant 5 & 30 & Lead & National & 40 \\
6 & Participant 6 & 250 & IT manager & National & 35 \\
7 & Participant 7 & 200 & Manager & National & 30 \\
8 & Participant 8 & 50 & Manager & & \\
\hline
\end{tabular}

4.2.1. Security Risk. Security risk was found to be the most common challenge to the adoption of cloud-based ERP around $14 \%$ of all the interviewees. Security is one of the core concerns, mentioned by most of our participants during the interview session. Perceived security is revealed mostly as a psychological issue aided by a lack of knowledge and trust. The participants mentioned that their organization and many other organizations expressed their major concerns about their data which is stored off-premises. This practice gives them a big sense of insecurity as well as a lack of control over their company data. Most organizations believe that their data can only be safe in their place and therefore are much reluctant to move toward cloud technology. They worried that their data may be stolen or leaked out during this implementation. Participant P2 of the interview session stated that "...Organization feel insecure about an information disclosure which company keeps hidden from the competitors in the market. Financial information is most of the stored data of any organization and is considered to be the heart of the company. Therefore, it is difficult for any organization to trust the 3rd party." Participants P3 and P6 mentioned that "... organizations are worried whether the cloud service provider will properly run the services or not." Furthermore, according to participant P5, “. . .security threat is not real for SMEs but just psychological issue. A large organization may have a concern about the real security threat while moving their data to the cloud." Participant P8 mentioned the cost problem, saying that "...ensuring the security is a responsibility of cloud service provider they charged for the service."

According to various participants, security concerns have more to do with human behavior and psychology rather than a real organizational issue. Participant P1 revealed that the "...security concerns that make organization reluctant about data transfer to the cloud, are mentality and psychological issues, it is not because of there is some kind of security problems but actually, the perception is negative, and they feel fear of threat." Participant P6 stated that the security of the cloud ERP system varies from vendor to vendor. Not all vendor provides sufficient security measures, not all vendors are ISO certified and not even made enough level of investment on the infrastructure. Furthermore, Participant P8 mentioned that “... the whole ERP security part deal with the service legal agreement (SLA). Many providers don't offer consistent and reliable services on security." He further explored the main reason for the data security issue is the lack of knowledge in this field and also not sufficient SLA, which makes cloud ERP fail.

From all these arguments and claims, it is concluded that the organizations are not much confident to adopt cloud ERP to secure their data. Some organizations, although adopted this technology but have concerns about data security as well as lack of control over data, which is still questioning by the organizations.

4.2.2. Performance. Performance was discussed in $7 \%$ of the selected interviews. Cloud ERP is one of the most online technology, requiring high-speed Internet sources. Participants P1, P3, and P6 indicated the performance of this system is the major challenge faced by cloud ERP implementation, accessed via the Internet. According to participant P1 "... delay in response time come because cloud ERP system run with a lot of processes as well as a huge amount of data to process online." This fact makes the cloud ERP much dependent on Internet availability and speed, suffering its 
performance. Further, he questioned that “... Is SQL server separate from application server? These resources directly impact application performance." You need to work with your IT team and cloud provider to ensure the servers running your applications have the proper resources for the number of users using that software. Participant P3 stated that ... "it is important to ensure that the activities and processes involved in cloud ERP would be able to work online efficiently. This fact can only be worked out if the cloud ERP system is made on modern architectures and the framework." He further pointed out that it is not necessary that all vendors made the same investment in the software to work over the Internet efficiently. The online efficiency of a system can only be increased if the system is based on modern technologies. All these problems make any organization reluctant to adopt ERP solutions. Participant P6 also added to this issue mentioning that " $\ldots$ the performance of ERP based system may depend on various factors, but the most important factor is the Internet connectivity because poor database performance slows down the whole system."

4.2.3. Customization. Customization is also a barrier towards the adoption of cloud-based ERP systems among the participants and reported 9\%. Customization being an issue for the cloud ERP system has been concisely mentioned by participants P1, P5, P6, and P7. According to Participant P1 and P6 "... although cloud-based ERPs offer many technological advantages, their customization is limited. In this case, if the customization is done, it charges the organization with a huge cost, affiliated with that specific change." Some vendors also allow their users to make changes and customize the system. In the case of small and medium enterprises, this practice is not much applicable as they do not have many resources to customize the system on their own because of a lack of skill and knowledge. As ask a question on the customization, according to participant P5, "...customization is important concerns which can be a barrier, organization need, and requirements change time by time you need a system that can easily be customized and not take much money on it. I suggest SME who can bear the cost at one time and keep relax will go for a hybrid model." The hybrid model allows organizations to protect their most valuable data on their terms; they are the ones who decide where important data are stored, and how that data are protected from external and internal threats. It puts control back in the best hands. In contrast to the participant (P7), "... When we said vendor to customize the solution then first they said that the system which we build is a generic system in which we apply best business practices according to the business needs and requirements, initially, we said ok when the system was deployed and accessed the needs and requirements are not too much similar as we doing before, because of not properly work on the needs and requirements of the organization into the meeting taken into the account with the vendor again this is happened because of lacking in knowledge." The customization of cloud ERP is limited but some of the vendors allow their clients if they want to customize the system, and they have the freedom to do but lack of skilled peoples brings the barriers to avail of that opportunity.

4.2.4. Internet Reliability. Internet reliability has been also reported by $9 \%$ of most of the interviewed participants. According to P1, P3, and P8, the cloud-based ERP mostly depends on the Internet in developing countries the reliability of Internet connectivity is a question. As asked from participants $\mathrm{P} 3$ and $\mathrm{P} 5$, the infrastructure of the network is not well facilitate as compare to developed countries, then people blame the system itself as slow whether then the problem lies with the Internet connectivity. In contrast to participants P1 and P8, “...traditional ERP does not rely on the Internet reliability but when we moved to cloud platform there will be a huge dependency over the Internet connectivity, so for this concern, we installed multiple Internet connections to cater." From all the above arguments, we believe that in Pakistan, there is a reliable solution to the Internet reliability organization which does not only rely on a single Internet service provider but also cloud ERP vendors can come with a different and better solution.

4.2.5. Lack of Awareness. Around 9\% of the participants mentioned lack of awareness as a significant barrier faced by an organization while adopting the cloud-based ERP. Participant P2 mentioned that "... How will we adapt if we do not know anything about cloud ERP so awareness is a challenge in Pakistani industry due to the lacking knowledge how to adopt system" and participant P4 declared that "... In Pakistan and our organization, we do not have much quality in education and have skilled people and knowledge about cloud ERP only a person in our organization who have little knowledge about this technology, Before moving to cloud ERP system one should be studied about the cloud ERP system, basic cloud knowledge is essential for the smoother adoption for the organization like SMEs, because they don't have much margin to make this adoption fail, this takes SMEs back." According to participant P5, “... Lack of awareness is an issue in Pakistan not everyone known about cloud ERP, IT training will be given to the head of the departments which will remove the hesitation to interact with the system" in addition to participant P3, “... We are not aware of cloud ERP our competitor pushes us to be aware of cloud ERP after that we are familiar with this new technology." From all concerns mentioned by participants how SMEs force to move towards enterprise solutions if they have no enough knowledge, enterprises are not well-informed benefits of cloud ERP, and they afraid to adopt due to negative perceptions. Therefore, a lack of awareness and knowledge left SMEs towards adoption.

4.2.6. In-House Resistance. User resistance is a challenge to the adoption of a cloud-based ERP system by organizations, approximately $19 \%$ of the selected participants. When an organization moves towards a cloud solution, in-house resistance to change from internal staff act like a suspending 
factor. Resistance to change is a natural thing that comes from internal staff because of not taking into consideration the selection process of the cloud ERP. Participant P1, P6, and P8 expressed the same "... When we shifted to a cloud ERP solution, there will be resistance from staff about their job security"; this is just because much IT-related work will be shifted to cloud ERP provider and employee feel the treat of their job. Participants P4, P3, and P5 said that “. . When a new system was adopted in the organization resistance come from the internal staff because they are lacking in knowledge and skills. And I want to add one thing very important don't keep an employee in the dark, communication with the employee in the workplace is always essential." When an organization is willing to adopt cloudbased ERP system, resistance occurred from the staff members, but this can be resolved by providing training to the employee and should be taken into consideration while adopting new technologies. In contrast to participants P2, P6, and P7 "... Resistance will come from staff end, they oppose to adopt, athletic training to the employee will remove uncertainty and after that, they are more comfortable to use such system more efficiently." Therefore, it appears that in-house resistance will come up from all our interviewee comments and claims resistance to change is a natural thing but can be resolved by taking employees into the selection process of ERP and provide some training to the employee by the organization.

4.2.7. Functionality Limitation. Functionality limitation was the least discussed factor and reported only $3 \%$ of the participants. Cloud ERP is a newbie in the world of technology, and enterprises still arise questions on its stability, reliability, and functionality. According to Participant P2 “... Cloud ERP are ready-made solutions, sometimes enterprise business process not mapped so the organization revised their business processes accordingly to that software" and added that further "... The ability to perform tasks and functionality still fear for many enterprises. Several enterprises are not very well aware and due to the lack of knowledge, they had a problem adopt smoothly. Cloud ERP functionality they think traditional ERP systems are old and mature, so time is taken to mature cloud ERP systems. However, some enterprises adopt cloud ERP and agreed their enterprises quickly shifted to the next level of advancement."

4.2.8. Vendor Trust. Vendor trust was discussed in 9\% of the participant's end. Participants P2 and P3 mentioned their concern about the ability and capability of local vendors. Vendors must provide support to their clients, but not enough support is provided thus some organizations preferred to build their solution. As stated by participant P6, the relationship of trust not only based upon vendor reputation but also on the agreement between them. In contrast to participant P7, he believes that "... Vendor trust is an issue because local vendor in here is not much mature, so NDA will sign with a vendor to our data should not be compromised and this is the responsibility of vendor." From all the above comments and discussion, vendor trust is a challenge when an organization adopts a cloud ERP system due to immature local vendors in the market.

4.2.9. External Pressure. External pressure is also a challenge towards the adoption of cloud-based ERP among organizations and reported 7\%. Previous studies on the adoption of technology define that external pressure is an indicator of adoption. In our study, when participants asked about the suggestion on the adoption of cloud ERP solution, P2, P3, and P6 believed that "... We cannot go to adopt new technology because of so many risk factors involved in it, when our competitors using the solution, so we are generally forced by different pressure to adopt new technologies" in addition to participant P6 “... In our surroundings technology is evolving very quickly and no other option to not adopt new technology, and especially when competitors use that technology, so it's become necessary to adopt due to competitive pressure." All participants during the interview agreed that they are forced to adopt new technologies by their competitor.

4.2.10. Lack of Knowledge. Lack of knowledge is a barrier towards the smooth adoption of cloud ERP solutions and reported 14\% from the participants. As stated by P1 and P6 "....Enterprise who decide to shift towards cloud may hinder while adoption because of less experience and knowledge." As declared by P3 and P4 that "...we will be in trouble because we don't have enough knowledge about the software.” Interviewee P5 “. . . Told us initially we are not aware of the cloud technology that is why we faced some challenges like security, customization, resistance, etc., lacking in knowledge is a big challenge and due to this we faced several challenges in adopting phase."

\section{Conclusion and Future Work}

SMEs are showing great interest in cloud ERP technology and are considered the best alternative rather than the traditional system; thus, it is essential to understand the challenges that can negatively impact the adoption in SMEs. In this regard, we identify ten (10) possible challenges of cloud ERP in the Pakistani context which influence the adoption decision. We used qualitative methodology along with unstructured interviews for extracting the challenges; and data collected from participants were successfully analyzed to ascertain the specific challenges. The study found challenges such as data security, customization limitation, external pressure, awareness, resistance to change, vendor competence, and lack of knowledge are the main problems to be mentioned when making a decision either to adopt or reject the cloud ERP systems. The reported work in this paper contributes to the area of information system adoption and especially in the domain of cloud ERP by identifying the challenges which impact small and medium enterprises. The research on cloud ERP systems required more exploration when it comes to the implications of cloud ERP, and much existing work focused on SMEs and did not 
interrogate whether the different size of enterprises relates to these implications. In this, we feel that we have at least begun to bridge the gap. Future research addresses this problem by comparing small- and large-sized enterprises and finds the difference in how they perceive cloud-based ERP.

\section{Data Availability}

The data collected during the data collection phase are available from the corresponding authors upon request.

\section{Conflicts of Interest}

The authors declare that they have no potential conflicts of interest.

\section{Acknowledgments}

This study was supported by the National Key Research and Development Plan (no. 2016YFC0303700), National Natural Science Foundation of China (no. 61972414), Beijing Natural Science Foundation (no. 4202066), Beijing Nova Program (no. Z201100006820082), and Fundamental Research Funds for Central Universities (nos. 2462020YJRC001 and 2462018YJRC040).

\section{References}

[1] K. H. Salum and M. Rozan, "Barriers and drivers in cloud ERP adoption among SMEs," Journal of Information Systems Research and Innovation, vol. 9, no. 1, pp. 9-20, 2015.

[2] T. N. Mahara, "Indian SMEs perspective for election of ERP in cloud," Journal of International Technology and Information Management, vol. 22, no. 1, p. 5, 2013.

[3] S. S. Shahawai and R. Idrus, "Malaysian SMEs perspective on factors affecting ERP system adoption," in Proceedings of the 2011 Fifth Asia Modelling Symposium, pp. 109-113, IEEE, Kuala Lumpur, Malaysia, May 2011.

[4] H. Klaus, M. Rosemann, and G. G. Gable, "What is ERP?" Information Systems Frontiers, vol. 2, no. 2, pp. 141-162, 2000.

[5] S. Fuller and T. S. McLaren, "Analyzing enterprise systems delivery modes for small and medium enterprises," in Proceedings of the 16th Americas Conference on Information Systems, AMCIS 2010, p. 380, Lima, Peru, August 2010.

[6] M. Armbrust, A. Fox, R. Griffith et al., "A view of cloud computing," Communications of the ACM, vol. 53, no. 4, pp. 50-58, 2010.

[7] J. Duan, P. Faker, A. Fesak, and T. Stuart, "Benefits and drawbacks of cloud-based versus traditional ERP systems," Proceedings of the 2012-13 course on Advanced Resource Planning, 2013.

[8] M. S. Amalnick, A. Ansarinejad, S.-M. Nargesi, and S. Taheri, "New perspective to ERP critical success factors priorities and causal relations under fuzzy environment," Journal of Mathematics and Computer Science, vol. 2, no. 1, pp. 160-170, 2011.

[9] H. U. Khan and H. S. I. A. Samad, "Enterprise strategic shift of technology: cloud-based systems verses traditional distributed system," International Journal of Enterprise Network Management, vol. 11, no. 4, pp. 320-346, 2020.

[10] A. Tahir, F. Chen, H. U. Khan et al., "A systematic review on cloud storage mechanisms concerning e-healthcare systems," Sensors, vol. 20, no. 18, p. 5392, 2020.
[11] R. Seethamraju and J. Seethamraju, "Adoption of ERPs in a medium-sized enterprise-a case study," ACIS 2008 Proceedings, vol. 104, Portland, OR, USA, May 2008.

[12] H. Samad and H. Khan, "Adoption of cloud in enterprises environment," in Proceedings of the 2017 DSI Annual Meeting, pp. 18-20, Washington, DC, USA, November 2017.

[13] R. Seethamraju, "Adoption of software as a service (SaaS) enterprise resource planning (ERP) systems in small and medium sized enterprises (SMEs)," Information Systems Frontiers, vol. 17, no. 3, pp. 475-492, 2015.

[14] S. A. Salim, "Cloud ERP adoption-a process view approach," in Proceedings of the PACIS, p. 281, Jeju Island, Korea, June 2013.

[15] G. C. A. Peng and C. Gala, "Cloud ERP: a new dilemma to modern organisations?" Journal of Computer Information Systems, vol. 54, no. 4, pp. 22-30, 2014.

[16] A. M. Albar and M. R. Hoque, "Factors affecting cloud ERP adoption in Saudi Arabia: an empirical study," Information Development, vol. 35, no. 1, pp. 150-164, 2019.

[17] A. H. Zadeh, B. A. Akinyemi, A. Jeyaraj, and H. M. Zolbanin, "Cloud ERP systems for small-and-medium enterprises," Journal of Cases on Information Technology, vol. 20, no. 4, pp. 53-70, 2018.

[18] U. M. Z. Usman, M. N. Ahmad, and N. H. Zakaria, "The determinants of adoption of cloud-based ERP of Nigerian's SMES manufacturing sector using toe framework and doi theory," International Journal of Enterprise Information Systems, vol. 15, no. 3, pp. 27-43, 2019.

[19] S. Ali, N. Ullah, M. F. Abrar, Z. Yang, and J. Huang, "Fuzzy multicriteria decision-making approach for measuring the possibility of cloud adoption for software testing," Scientific Programming, vol. 2020, Article ID 6597316, 24 pages, 2020.

[20] A.-S. Moh'd Anwer, "Towards better understanding of determinants logistical factors in SMEs for cloud ERP adoption in developing economies," Business Process Management Journal, vol. 25, no. 5, pp. 887-907, 2019.

[21] S. Tongsuksai, S. Mathrani, and N. Taskin, "Cloud enterprise resource planning implementation: a systematic literature review of critical success factors," in Proceedings of the 2019 IEEE Asia-Pacific Conference on Computer Science and Data Engineering (CSDE), pp. 1-8, IEEE, December 2019, Melbourne, Australia.

[22] B. Ahn and H. Ahn, "Factors affecting intention to adopt cloud-based ERP from a comprehensive approach," Sustainability, vol. 12, no. 16, p. 6426, 2020.

[23] S. Ali and H. Li, "Moving software testing to the cloud: an adoption assessment model based on fuzzy multi-attribute decision making algorithm," in Proceedings of the 2019 IEEE 6th International Conference on Industrial Engineering and Applications (ICIEA), pp. 382-386, IEEE, Tokyo, Japan, April 2019.

[24] R. Small, "Factors affecting the adoption of enterprise resource planning (ERP) on cloud among small and medium enterprises (SMES) in Penang, Malaysia," Journal of Theoretical and Applied Information Technology, vol. 88, no. 3, 2016.

[25] E. O. Yeboah-Boateng and K. A. Essandoh, "Factors influencing the adoption of cloud computing by small and medium enterprises in developing economies," International Journal of Emerging Science and Engineering, vol. 2, no. 4, pp. 13-20, 2014.

[26] K. H. Salum and M. Rozan, "Exploring the challenge impacted SMEs to adopt cloud ERP," Indian Journal of Science and Technology, vol. 9, no. 45, pp. 1-8, 2016. 
[27] J. Rajapakse and P. Seddon, "ERP adoption in developing countries in Asia: a cultural misfit," in Proceedings of the 28th Information Systems Seminar in Scandinavia, pp. 6-9, Kirstiansand, Norway, 2005.

[28] M. Saunders, P. Lewis, and A. Thornhill, Research Methods for Business Students, Pearson Education Limited, Harlow, UK, 2009.

[29] A. Alajbegovic, V. Alexopoulos, and A. Desalermos, Factors influencing cloud ERP adoption: a comparison between SMES and large companies, 2013.

[30] S. Kvale and S. Brinkmann, Interviews: Learning the Craft of Qualitative Research Interviewing, Sage, Thousand Oaks, CA, USA, 2009.

[31] M. Bengtsson, "How to plan and perform a qualitative study using content analysis," NursingPlus Open, vol. 2, pp. 8-14, 2016.

[32] V. Minichiello, R. Aroni, and V. Minichiello, In-Depth Interviewing: Researching People, Longman Cheshire, Harlow, UK, 1990. 